\title{
Thermo-Switchable Janus Gold Nanoparticles with Stimuli-Responsive Hydrophilic Polymer Brushes
}

Xiaoqin Niu ${ }^{\mathrm{a}}$, Fen Ran ${ }^{\mathrm{a}, \mathrm{b}, *}$, Limei Chen ${ }^{\mathrm{a}}$, Gabriella Jia-En Lu ${ }^{\mathrm{a}}$, Peiguang $\mathrm{Hu}^{\mathrm{a}}$, Christopher P. Deming $^{\mathrm{a}}$, Yi Peng ${ }^{\mathrm{a}}$, Mauricio D. Rojas-Andrade ${ }^{\mathrm{a}}$, and Shaowei Chen ${ }^{\mathrm{a}, *}$

${ }^{a}$ Department of Chemistry and Biochemistry, University of California, 1156 High Street, Santa Cruz, California 95064, USA

${ }^{\mathrm{b}}$ State Key Laboratory of Advanced Processing and Recycling of Non-ferrous Metals, Lanzhou University of Technology, Lanzhou 730050, P. R. China

*Corresponding authors: Fen Ran (ranfen@163.com); Shaowei Chen (haowei@ucsc.edu)

Table S1. Summary of molecular information of the synthesized $\mathrm{PMEO}_{2} \mathrm{MA}-\mathrm{CMTB}$ polymers

\begin{tabular}{|c|c|c|c|c|}
\hline $\begin{array}{c}\text { Monomer to } \\
\text { RAFT agent ratio } \\
\text { (wt./wt.) }\end{array}$ & $10: 1$ & $30: 1$ & $60: 1$ & $150: 1$ \\
\hline $\mathrm{M}_{\mathrm{n}}\left(\times 10^{3}\right)$ & 1.5 & 4.8 & 10.2 & 30.4 \\
\hline $\mathrm{M}_{\mathrm{w}} / \mathrm{M}_{\mathrm{n}}$ & 1.21 & 1.24 & 1.25 & 1.33 \\
\hline
\end{tabular}

Table S2. Summary of FTIR data of the synthesized $\mathrm{PMEO}_{2} \mathrm{MA}$ polymers

\begin{tabular}{|c|c|}
\hline $\begin{array}{c}\text { Peak positions } \\
\left(\mathrm{cm}^{-1}\right)\end{array}$ & Assignments \\
\hline 2940 & Methyl and methylene $\mathrm{C}-\mathrm{H}$ stretches \\
\hline 2654 & $\mathrm{~S}-\mathrm{H}$ stretch \\
\hline 1740 & $\mathrm{C}=\mathrm{O}$ stretch \\
\hline 1456 & $\mathrm{CH}_{2}$ bending \\
\hline 1250 & $\mathrm{CH}_{2}-\mathrm{O}$ stretch \\
\hline 1144 & $\mathrm{CH}_{2}-\mathrm{O}$ stretch \\
\hline
\end{tabular}

Table S3. Summary of FTIR data of the $\mathrm{PMEO}_{2} \mathrm{MA}$-tethered gold Janus nanoparticles

\begin{tabular}{|c|c|}
\hline $\begin{array}{c}\text { Peak positions } \\
\left(\mathrm{cm}^{-1}\right)\end{array}$ & Assignments \\
\hline 2940 & Methyl and methylene $\mathrm{C}-\mathrm{H}$ stretches \\
\hline 1740 & $\mathrm{C}=\mathrm{O}$ stretch \\
\hline 1250 & $\mathrm{CH}_{2}-\mathrm{O}$ stretch \\
\hline
\end{tabular}

